\title{
ANÁLISE DO PERFIL DO PACIENTE COMO VÍTIMA DE MÚLTIPLOS TRAUMAS*
}

\author{
Kátia Cilene Godinho Bertoncello, Cibele D’Avila Kramer Cavalcanti², Patrícia Ilha²
}

\begin{abstract}
RESUMO: Estudo quantitativo, exploratório, descritivo e transversal foi realizado na unidade de emergência de hospital referência no atendimento de múltiplos traumas. Objetivou-se analisar o perfil dos pacientes acometidos por múltiplos traumas. A coleta de dados ocorreu de fevereiro a abril de 2012, a amostra se constituiu de 41 pacientes, maiores de 18 anos, de ambos os sexos, não gestantes e que deram entrada por meio de serviço de atendimento pré-hospitalar. Houve predomínio de vítimas do sexo masculino (82,9\%), com idade entre 18 e 30 anos (36,5\%), em sua maioria motociclistas $(51,2 \%)$ e que apresentaram alterações leves nas funções vitais. Concluiu-se que se deve conhecer o perfil do cliente atendido a fim de preparar a dinâmica do serviço, considerando o planejamento um instrumento essencial para a assistência, principalmente no que diz respeito à vítima de múltiplos traumas, a qual requer atendimento ágil e especializado.
\end{abstract}

PALAVRAS-CHAVE: Traumatismo múltiplo; Enfermagem em emergência; Serviço hospitalar de emergência.

\section{ANALYSIS OF THE PROFILE OF THE PATIENT AS VICTIM OF MULTIPLE TRAUMA}

ABSTRACT: This quantitative, exploratory, descriptive and cross-sectional study was undertaken in the Emergency Department of a hospital specialized in treating multiple trauma. The aim was to analyze the profile of the patients affected by multiple trauma. Data collection took place in February-April 2012, and the sample comprised 41 patients, over 18 years of age, of both sexes, not pregnant, and who came via the pre-hospital care service. The majority of victims were male (82.9\%), aged between 18 and 30 (36.5\%), motorcyclists (51.2\%) and presented mild alterations in vital functions. It was concluded that it is necessary to investigate the profile of the client attended in order to prepare the dynamics of the service, considering the planning of an instrument to be essential for the care, principally concerning the victim of multiple trauma, who requires rapid and specialized care.

KEYWORDS: Multiple trauma; Emergency nursing; Emergency hospital service.

\section{ANÁLISIS DEL PERFIL DEL PACIENTE COMO VÍCTIMA DE MÚLTIPLOS TRAUMAS}

RESUMEN: Estudio cuantitativo, exploratorio, descriptivo y transversal realizado en la unidad de emergencia de hospital referencia en el atendimiento de múltiplos traumas. El objetivo fue analizar el perfil de los pacientes víctimas de múltiplos traumas. Los datos fueron obtenidos de febrero a abril de 2012 y la muestra se constituye de 41 pacientes, mayores de 18 años, de ambos los sexos, no gestantes y que entraron por medio de servicio de atendimiento prehospitalar. Hubo predominio de víctimas del sexo masculino (82,9\%), con edad entre 18 y 30 años (36,5\%), en su mayoría motociclistas $(51,2 \%)$ y que presentaron alteraciones sutiles en las funciones vitales. Se concluye que es necesario conocer el perfil del cliente atendido para preparar la dinámica del servicio, considerando el planeamiento de un instrumento esencial para la asistencia, principalmente con relación a la víctima de múltiplos traumas, la cual necesita atendimiento ágil y especializado.

PALABRAS CLAVE: Traumatismo múltiplo; Enfermería en emergencia; Servicio hospitalar de emergência.

*Extraído do Trabalho de Conclusão de Curso de Graduação em Enfermagem da Universidade Federal de Santa Catarina - UFSC intitulado Cuidados de enfermagem ao paciente vítima de múltiplos traumas: diagnósticos e propostas de intervenções, baseados em NANDA e NIC apresentado em 2012

${ }^{1}$ Enfermeira. Doutora em Enfermagem Fundamental. Professora do Departamento de Enfermagem da UFSC. Coordenadora do Mestrado Profissional Associado a Residência Multidisciplinar em Saúde - UFSC. Pesquisadora do Grupo de Estudo no Cuidado de Pessoas nas Situações Agudas de Saúde - GEASS.

${ }^{2}$ Enfermeira. Membro do GEASS.

Autor correspondente:

Recebido: $29 / 07 / 2012$

Katia Cilene Godinho Bertoncello

Aprovado: 21/10/2012

Universidade Federal de Santa Catarina

Rua Hipólito Mafra, 203 - 88045-410 - Florianópolis-SC-Brasil

E-mail:kbertoncello@yahoo.com.br 


\section{INTRODUÇÃO}

Os múltiplos traumas incorrem em danos sistêmicos significativos, como falha ou disfunção de órgãos vitais $^{(1-2)}$. No atendimento, o enfermeiro é responsável pela prestação de cuidado ao paciente de acordo com suas prioridades e, no sistema hospitalar, tem como atribuição a realização dos diagnósticos e prescrições dos cuidados de enfermagem, principalmente no que diz respeito à vítima de múltiplos traumas, com o objetivo de reduzir os danos e potencializar o tratamento ${ }^{(3)}$.

De acordo com a Classificação Internacional de Doenças, a partir de 1980 as causas externas são consideradas: acidentes de transporte, lesões autoprovocadas intencionalmente, agressões, intervenções legais e operações de guerra. Essas causas sofreram considerável aumento, alterando o perfil epidemiológico brasileiro, visto que passaram a representar a segunda causa de morte na perspectiva geral e primeira na faixa etária entre cinco e $39 \operatorname{anos}^{(4)}$. No ano de 2010, ocorreram 40.610 mortes fatais no trânsito brasileiro, sendo $25 \%$ desses relacionados a motociclistas, chegando a 145 mil internações no Sistema Único de Saúde. Em Santa Catarina ocorreram 1.862 óbitos, entre os quais 557 decorrentes de acidentes motociclisticos ${ }^{(5)}$.

A unidade de Urgência e Emergência deve estar pronta para atender o paciente vítima de trauma. O planejamento é um instrumento essencial para assistência à vida. Ter uma equipe de saúde treinada e coordenada nas ações e equipamentos disponíveis influencia na sobrevida do atendimento à vítima de trauma ${ }^{(6)}$. Para realizar esse cuidado integral, otimizado e ágil, os enfermeiros devem conhecer o perfil do cliente atendido em sua área de abrangência e referência, a fim de preparar a dinâmica do atendimento. Baseado neste princípio e na existência desta lacuna de conhecimento, para esta região do Brasil, justificou-se a realização desta investigação que teve como objetivo analisar o perfil do paciente acometido de múltiplos traumas atendido em um hospital estadual, cuja Unidade de Emergência é referência regional para traumatologia e ortopedia.

\section{MÉTODO}

Trata-se de um estudo de abordagem quantitativa, exploratória, descritiva e transversal, realizado na Unidade de Emergência de um Hospital Estadual público, o qual é referência regional para traumatologia e ortopedia e está localizado em Florianópolis-Santa Catarina.
A amostra constitui-se de 41 pacientes atendidos na Unidade de Emergência, no período de 17 de fevereiro a 6 de abril de 2012. Todos foram encaminhados pelo serviço de atendimento pré-hospitalar. Os critérios de inclusão foram: ser paciente maior de 18 anos, que não se encontrasse gestantes, com Escala de Coma de Glasgow maior ou igual a três, e avaliado pelas pesquisadoras no momento da entrada no hospitalar.

O instrumento utilizado para a coleta de dados foi a ficha de atendimento utilizado pelo serviço de resgate pré-hospitalar da Policia Rodoviária Federal. Esta foi adaptada de acordo com atendimento no nível de média complexidade e abordagem do paciente na unidade intra-hospitalar. A ficha abrangeu seis campos: identificação, avaliação dos sinais vitais, avaliação neurológica, levantamento do passado médico, avaliação das lesões e avaliação da cinemática do trauma. Os dados coletados foram analisados pelo programa estatístico Statistical Package for the Social Sciences - SPSS ${ }^{\circledR}$, versão 17.

O estudo foi aprovado pelo Comitê de Ética e Pesquisa com Seres Humanos da Pró-Reitoria de Pesquisa e Extensão da Universidade Federal de Santa Catarina, (n. CAAE 482783) e pelo Comitê de Ética e Pesquisa em Seres Humanos do Hospital Governador Celso Ramos (n. 2012/0001). Todos os participantes foram convidados a participar da investigação após a finalização do atendimento. Foi esclarecido o objetivo do estudo, dado garantia do anonimato e liberdade de desistência a qualquer momento. Frente ao aceite, foi apresentado o Termo de Consentimento Livre e Esclarecido, o qual todos assinaram.

\section{RESULTADOS}

A caracterização do perfil dos pacientes atendidos está apresentado nas tabelas 1 a 5 à seguir.

Tabela 1 - Fatores que influenciaram os pacientes acometidos de múltiplos traumas. Florianópolis, 2012

\begin{tabular}{lcc}
\hline \multicolumn{1}{l}{ CONSUMO DE BEBIDA } & ALCOÓLICA \\
\hline Sim & 6 & $14,6 \%$ \\
Não & 35 & $85,4 \%$ \\
Total & 41 & $100 \%$ \\
\hline UTILIZAÇÃO DE DROGAS ILÍCITAS \\
\hline Sim & 2 & $4,8 \%$ \\
Não & 39 & $95,2 \%$ \\
Total & 41 & $100 \%$ \\
\hline
\end{tabular}


Tabela 2 - Caracterização dos pacientes e dos acidentes acometidos de múltiplos traumas $(\mathrm{N}=41)$. Florianópolis, 2012

\begin{tabular}{|c|c|c|}
\hline CARACTERÍSTICAS & $\mathbf{N}$ & $\%$ \\
\hline \multicolumn{3}{|l|}{ SEXO } \\
\hline Masculino & 34 & 83,0 \\
\hline Feminino & 7 & 17,0 \\
\hline \multicolumn{3}{|c|}{ FAIXA ETÁRIA (EM ANOS) } \\
\hline $18-30$ & 15 & 36,5 \\
\hline $31-40$ & 11 & 26,9 \\
\hline $41-50$ & 6 & 14,7 \\
\hline$<51$ & 9 & 21,9 \\
\hline \multicolumn{3}{|c|}{ LOCAL DA VÍTIMA NA HORA DO ACIDENTI } \\
\hline Automóvel & 5 & 12,3 \\
\hline Motocicleta & 21 & 51,3 \\
\hline Bicicleta & 1 & 2,4 \\
\hline Via Pública (Pedestre) & 7 & 17,0 \\
\hline Outros & 7 & 17,0 \\
\hline \multicolumn{3}{|c|}{ MECANISMO DO TRAUMA } \\
\hline Automóvel & 19 & 46,3 \\
\hline Caminhão & 2 & 4,8 \\
\hline Motocicleta & 2 & 4,8 \\
\hline Anteparo & 3 & 7,2 \\
\hline Outros & 7 & 17,0 \\
\hline Ferimento por arma branca & 2 & 4,9 \\
\hline Queda de nível & 6 & 15 \\
\hline \multicolumn{3}{|l|}{ TIPO DE COLISÃO } \\
\hline Frontal & 17 & 41,4 \\
\hline Traseira & 2 & 4,8 \\
\hline Lateral & 6 & 14,5 \\
\hline Capotamento & 3 & 7,3 \\
\hline Queda de nível & 3 & 7,3 \\
\hline Atropelamento & 1 & 2,4 \\
\hline Outros & 1 & 2,4 \\
\hline Não informado & 8 & 19,9 \\
\hline
\end{tabular}

Tabela 3 - Área corporal lesada em pacientes acometidos de múltiplos traumas $(\mathrm{N}=41)$. Florianópolis, 2012

\begin{tabular}{lcc}
\hline LOCAL DO TRAUMA & $\mathbf{N}^{\mathbf{0}}$ & $\mathbf{\%}$ \\
\hline Membros inferiores & 25 & 28,4 \\
Membros Inferiores & 18 & 20,4 \\
Face & 13 & 14,7 \\
Coluna & 7 & 7,9 \\
Arcos Costais & 6 & 6,8 \\
Pelve & 2 & 2,2 \\
Órgãos Internos & 1 & 1,5 \\
\hline *Mais de uma área corporal lesada na amostra do estudo &
\end{tabular}

Tabela 4 - Alterações fisiológicas sofridas pelos pacientes acometidos de múltiplos traumas $(\mathrm{N}=41)$. Florianópolis, 2012

\begin{tabular}{lcc}
\hline ALTERAÇÕES & N & \% \\
\hline RESPIRATÓRIAS & 27 & 65,8 \\
Eupnéia & 11 & 26,8 \\
Taquipnéia & 3 & 7,4 \\
Bradipnéia & & \\
SATURAÇÃO DE OXIGÊNIO & 41 & 100 \\
$>86 \%$ & & \\
FREQUÊNCIA CARDÍACA & 23 & 56,0 \\
Taquicardia & 15 & 36,6 \\
Eucardia & 3 & 7,4 \\
Bradicardia & & \\
PRESSÃO ARTERIAL & 20 & 48,8 \\
Normal & 19 & 46,3 \\
Hipertensão & 2 & 4,9 \\
Hipotensão & & \\
TEMPERATURA CORPORAL & 30 & 73,2 \\
Normal & 11 & 26,8 \\
$<36,4^{\circ} \mathrm{C}$ & & \\
CARACTERÍSTICA DA PELE & 3 & 7,4 \\
Úmida $_{\text {Seca }}$ & 38 & 92,6 \\
\hline
\end{tabular}

Tabela 5 - Alterações neurológicas apresentadas pelos pacientes acometidos de múltiplos traumas $(\mathrm{N}=41)$. Florianópolis, 2012

\begin{tabular}{lcc}
\hline ESCALA DE COMA DE GLASGOW & N & $\mathbf{\%}$ \\
\hline 3 & 4 & 9,7 \\
$4-8$ & 1 & 2,5 \\
$9-12$ & 2 & 5,0 \\
$13-15$ & 34 & 82,9 \\
PUPILAS & & \\
Isocóricas & 34 & 82,9 \\
Anisocóricas & 3 & 7,3 \\
Midriáticas & 2 & 4,8 \\
Média fixas & 1 & 2,5 \\
Mióticas & 1 & 2,5 \\
REAGENTES & & \\
Sim & 37 & 90,2 \\
Não & 4 & 9,8 \\
\hline
\end{tabular}




\section{DISCUSSÃO}

Nas últimas décadas, o número de acidentes de trânsito vem crescendo substancialmente devido ao aumento no número de veículos, imprudência dos motoristas, falta de fiscalização por parte das autoridades, aumento do consumo de álcool e drogas e o dinamismo cotidiano. As características socioeconômicas, culturais e políticas influenciam na causa das ocorrências ${ }^{(7)}$. A maioria dos pacientes do presente estudo corrobora com outros estudos que traçam o perfil de pacientes em diversas regiões do país. Estudo realizado no Estado do Paraná, entre 3.871 vítimas, predominaram adultos jovens entre 20 e 29 anos (1.279 - 33\%) e condutores motociclistas (1.603 - 41,6\%). No Estado de Minas Gerais, dentre os 82 pacientes pesquisados, a principal faixa etária acometida foi jovens entre os 20 e 29 anos, com total de $32(35,1 \%)$, sendo motociclistas 37 $(40,7 \%)$ e do sexo masculino $53(58,2 \%)^{(8)}$.

A predominância das vítimas do gênero masculino é um padrão que se repete no perfil brasileiro. Dos fatores possíveis, e que se relacionam com esse trabalho, aquele que pode estar mais fortemente envolvido com as causas de trauma é o cultural. Homens frequentemente são condutores de carro e/ou moto, possuem ambas as habilitações e aprendem a dirigir com menor idade. $\mathrm{O}$ gênero feminino apresenta maior cautela no trânsito, dirige com menos frequência e apresenta menor representatividade no número de carteiras de habilitação ${ }^{(9)}$. Indicadores brasileiros trazem que homens têm 12 vezes mais chances de morrer por causas traumáticas que mulheres ${ }^{(10)}$.

Referente à idade, há de adultos jovens, entre 18 e 30 anos. Características da faixa etária, como imaturidade, inexperiência ao volante, tendência de superestimar a capacidade, além de outros comportamentos de risco, como iniciação precoce da ingesta de bebidas alcoólicas e contato maior com substâncias ilícitas, são alguns fatores que podem ter uma forte ligação com as estatísticas ${ }^{(11)}$.

A utilização de drogas e álcool agrava este quadro. Resultados relacionam o elevado índice de acidentes com a utilização destas substâncias ${ }^{(12)}$, as quais alteram os níveis cognitivos de forma a reduzir as habilidades motoras e sensoriais ${ }^{(8,13)}$. Constatamos que entre os pacientes investigados, $6(14,6 \%)$ fizeram uso de álcool e $2(4,8 \%)$ de drogas ilícitas . Há a necessidade de investigar a natureza e as crenças deste público e trabalhar, educacionalmente, para alterar esse padrão de risco ao dirigir.
Uma barreira para levantamento real de dados é o elevado número de óbitos envolvendo jovens ${ }^{(10)}$. Os motociclistas são os mais atingidos pela epidemia do trauma. No Brasil, só de janeiro a março de 2012, foram constatados 238 óbitos $^{(4)}$. Ainda, há de se levar em consideração o grande número de motos circulantes; meio de transporte de baixo custo, relativo à aquisição $\mathrm{e}$ manutenção ${ }^{(8)}$, o que contribui para o aumento da frota.

Um fator que pode estar relacionado entre esta modalidade de transporte e o trauma é a dinâmica de funcionamento e estrutura, a qual não apresenta um sistema de proteção adequado, de forma que qualquer acidente pode trazer graves consequências ao motociclista. Além dos hábitos de imprudência e desrespeito à legislação, é grande o número de motociclistas que transitam entre os carros, pelos acostamentos e vias perigosas; comportamentos de riscos que aumentam as probabilidades de ocorrência de acidentes bem como impõem perigo a outros condutores e pedestres. Contudo, neste estudo foi encontrado apenas um $(2,4 \%)$ atropelamento causado por moto.

Dentre os outros motivos de causa de trauma, o que se apresentou mais relevante neste estudo foi a queda de nível, considerada importante e de características graves, pois, dependendo do desnível, associado à velocidade e à queda, os risco de lesões se agravam de acordo com a cinemática do acidente. As principais lesões constatadas nessa causa foram: queda em pé (membros inferiores, pelve e coluna torácica); queda para frente (punhos); queda de cabeça (traumatismo crânio encefálico e de coluna cervical). No caso de não haver um sentido específico, deve-se avaliar a parte do corpo que tocou inicialmente o chão ${ }^{(14)}$.

É muito importante o levantamento dos dados dos pacientes, assim como um breve histórico da ocorrência, a fim de conhecer a cinemática do trauma, possíveis comorbidades, entre outros fatores, que possam causar alterações significantes para sua estabilidade vital. Além disso, torna-se necessário a realização de um exame físico integral com uma avaliação objetiva, ágil e eficiente ${ }^{(6)}$.

No que tange ao tipo de colisão, deve ser levada em consideração a origem do impacto e a perspectiva da colisão, de forma que cada característica pode exercer uma força e uma dispersão energética específica. Isso pode afetar, diferentemente, cada sistema, causando lesões dos mais diferentes graus. Neste estudo as colisões frontais foram mais incidentes $17(41,4 \%)$; na trajetória do impacto frontal o ocupante, geralmente, assume o trajeto: ou por cima ou por baixo. Naquele, 
a vítima é exposta, principalmente, a lesões de órgãos internos, como fígado, baço e intestino, lesão de coluna cervical, traumatismo crânio encefálico e rompimento de grandes vasos, como a aorta no segmento abdominal e torácico. Já no último, os ricos maiores são fraturas e ferimentos em membros inferiores, rompimento de grandes vasos, como artéria poplítea, e traumatismo craniano $^{(14)}$.

Os sinais vitais são fortes indicadores de que alguma função vital pode estar comprometida. Por meio dos sinais vitais pode-se avaliar funções hemodinâmicas, respiratórias e termorreguladoras, que são as essenciais para manutenção da vida. Ressalta-se a importância da avaliação neurológica, a qual pode afetar diretamente as funções vitais ${ }^{(3)}$. Neste estudo as principais alterações foram relativas ao sistema respiratório; 11 $(26,8 \%)$ pacientes apresentaram-se taquipneicos. Esse sinal pode representar diversas alterações sistêmicas, a mais importante delas, e passível de evoluir para um quadro mais grave, é a insuficiência respiratória. Essa pode ser motivada também por doenças clínicas de base respiratórias ou alterações do sistema nervoso central, resultantes indiretamente de traumas, drogas ou patogenias ${ }^{(3,6,14)}$.

Um importante fator que pode motivar a alteração no sistema respiratório é o estado psicológico em que a vítima se encontra. Considerando-se a exposição do paciente a uma circunstância de grande nível de estresse em um ambiente desconhecido, recebendo, a todo o momento, novas informações, tendo seu corpo manipulado e exposto, há ação imediata do organismo, ativando o sistema simpático ${ }^{(3,6,14-15)}$.

Quanto à saturação de oxigênio, que nos mostra os níveis de oxigênio carreados pela hemoglobina presente no sangue arterial, a verificação desta alteração não foi verificada, visto que grande parte das vítimas já chegavam aohospital com aporte de oxigênio e apresentavam uma saturação dentro dos níveis aceitáveis. Para as alterações de origem circulatória, 23 (56\%) vítimas apresentaram taquicardia e 19 (46,3\%) elevação da pressão arterial, podendo ter como causa a alteração psicológica da exposição ao ambiente e situação de estresse. A taquicardia e elevação da pressão arterial podem ter origem por diversos fatores ${ }^{(6)}$, todavia, deve-se estar atento aos principais fatores, quando se trata de vítima de múltiplos traumas. Alterações hemodinâmicas podem ser indícios de fase inicial de choque hipovolêmico, causado por grande sangramento, aparente ou não (lesão de órgãos internos), trauma crânioencefálico, traumatismo de coluna, exposição intensa a dor, entre outros fatores ${ }^{(6,14)}$.

A temperatura corporal, quando dentro dos parâmetros de normalidade $\left(36,4^{\circ}\right.$ a $\left.37,6^{\circ} \mathrm{C}\right)$ indica melhor funcionamento celular e tecidual do organismo humano. De acordo com a idade, a temperatura pode ter alternâncias, visto a diferença entre o metabolismo celular em cada fase da vida ${ }^{(16)}$. Outros fatores externos podem alterar seu padrão, como doenças clínicas, alterações de temperatura do ambiente, alterações oxi-hemodinâmicas e traumas. Essas variações, mesmo que moderadas, acarretam em alterações importantes ao organismo. Entre os pacientes deste estudo, 11 $(26,8 \%)$ apresentaram hipotermia, alteração que acarreta instabilidade e risco de agravos, como arritmias cardíacas, baixo débito cardíaco, infecção, doenças provenientes de alterações de coagulação sanguínea e baixa perfusão tissular( ${ }^{(17)}$.

Características como a umidade e a temperatura ao toque podem ajudar a definir as causas e as condutas a serem tomadas. Neste estudo, a maioria dos pacientes apresentou pele seca (38 - 92,6\%) e morna ( 37 - 90,2\%), o que pode representar que alterações de temperatura ocorreram por fatores de estresse, tentativa do organismo em restabelecer as funções, tentativa de impedir processos inflamatórios ou por estímulos dolorosos ${ }^{(18)}$.

Outra avaliação essencial é a neurológica, na qual se estabelece o nível de consciência da vítima, assim como o tamanho das pupilas e a sua reação. Como instrumento desta avaliação usou-se a Escala de Coma de Glasgow (ECG). O rebaixamento do nível de consciência pode representar a diminuição da oxigenação e/ou perfusão cerebral, ou ser resultado de um trauma direto ao cérebro ${ }^{(6)}$. Neste estudo, os pacientes que representaram grave rebaixamento, com escore da ECG igual a três, apresentavam-se em intubação orotraqueal, e sobre o efeito de sedativos, o que interfere nesta avaliação. Porém, a maioria dos pacientes (34 - 82,9\%) apresentou boa resposta neurológica, com Escore de Coma de Glasgow entre 13 e 15. Quanto às pupilas, suas alterações podem também estar diretamente relacionadas, não apenas às condições patológicas, mas ao uso de substâncias alcoólicas e drogas e por estado psicológico alterado ${ }^{(6,18)}$, percebidos em apenas $2(4,8 \%)$ participantes.

Quando analisamos as áreas onde ocorreram as lesões, as principais foram: membros inferiores, membros superiores, crânio e face, o que corrobora com os dados do tipo de colisão que foram em maior número, frontais e de acordo com a cinemática do trauma, visto que essas regiões são as principalmente afetadas ${ }^{(14)}$. É 
nos membros inferiores que se encontram os ossos mais longos do corpo, como o fêmur, a tíbia e a fíbula, deste modo, quando a energia da colisão é dissipada, são essas estruturas que absorvem maior impacto, resultando, principalmente, em fraturas. Já os membros superiores, na maioria das vezes, são utilizados de forma reflexa como uma proteção à vítima. Ela os utiliza como uma forma de amenizar o impacto de outras partes do corpo, resultando em fraturas, entorses e escoriações ${ }^{(6)}$.

\section{CONCLUSÃO}

A epidemiologia do trauma é um fator em destaque cotidiano e destaca-se a relevância em se investir em estudos, campanhas e políticas nacionais de redução de morbi-mortalidade contribuindo para melhor assistência aos pacientes e melhor qualidade de vida.

É importante conhecer o perfil de pacientes vítimas de múltiplos traumas para também preparar a equipe de saúde e articular os diversos setores, com vistas a elaborar protocolos de cooperação na prevenção de acidentes assim como instrução para capacitação dos profissionais de saúde de acordo com as diretrizes. Ao conhecer o perfil das vítimas também é possível melhor dispor dos recursos, otimizando o serviço e tornando-o mais ágil.

\section{REFERÊNCIAS}

1. Steinman M, Figueiredo LFP, Ramalho Júnior A. Politraumatismo. In: Knobel E. Condutas ao paciente grave. $3^{\text {a }}$ ed. São Paulo: Atheneu; 2006.

2. Ministério da Saúde (BR). Portaria n ${ }^{\circ}$ 2.048/GM/MS, de 5 de novembro de 2002. Institui o Regulamento Técnico dos Sistemas Estaduais de Urgência e Emergência. 2002.

3. Bare BG, Suddarth DS. Tratado de enfermagem médico-cirúrgica. 12a ed. Rio de Janeiro: Guanabara Koogan; 2011.

4. Ministério da Saúde (Brasil). Acidentes de trânsito matam 1.862 em SC. Brasília, 2002. [acesso em 20 fev 2012] Disponível: http://portalsaude.saude.gov.br/ portalsaude/noticia/2955/162/acidentes-de-transitomatam-1.862-pessoas-em-sc.html.

6. Ministério da Saúde (BR). Departamento de Informática do SUS. Brasília, 2012 [acesso 25 fev 2012]. Disponível: http://www2.datasus.gov.br/DATASUS/index. php?area $=0201$
7. ATLS. Advanced trauma life support. 8th ed. American College of Surgeons. Chicago: Ilinois; 2008.

8. Andrade SM, Soares DA, Braga GP, Moreira JH, Botelho FMN. Comportamentos de risco para acidentes de trânsito: um inquérito entre estudantes de medicina na região sul do Brasil. AMB Rev. Assoc. Med. Bras. [Internet] 2003;49(4) [acesso 25 fev 2012]. Disponível: http://www.scielo.br/scielo.php?script=sci arttext\&pid=S0104-42302010000500013\&lng=en\&n $\mathrm{rm}=\mathrm{iso}>$. ISSN 0104-4230. http://dx.doi.org/10.1590/ S0104-42302010000500013.

9. Freire E. Trauma: a doença dos séculos. São Paulo: Atheneu; 2001.

10. Ferreira TFA, Nápolis ACR, Lima CS, Araújo LC, Garcia CB, Lima PS, et al. Estudo da gravidade dos pacientes vítimas de acidentes de trânsito atendidos pelo hospital de clínicas de Uberlândia de dezembro de 2005 a março de 2006 segundo índices de trauma. Biosci. J. 2009;25(2):152-60.

11. Ministério da Saúde (BR). Painel de indicadores do SUS. Departamento de Monitoramento e Avaliação da Gestão do SUS. Secretaria de Gestão Estratégica e Participativa. Ano I, nº 1. 2006.

12. Sauer MTN, Wagner MB. Acidentes de trânsito fatais e sua associação com a taxa de mortalidade infantil e adolescência. Cad. Saúde Pública. [Internet] 2003;19(5) [acesso 25 fev 2012]. Disponível: http://www.scielo.br/ pdf/csp/v19n5/17824.pdf

13. Marín L, Queiroz MS. A atualidade dos acidentes de trânsito na era da velocidade: uma visão geral. Cad. Saúde Pública. [Internet] 2000;16(1) [acesso $25 \mathrm{fev}$ 2012]. Disponível: http://www.scielosp.org/pdf/csp/ v16n1/1560.pdf

14. Koizumi MS. Acidentes de motocicleta no município de São Paulo, SP (Brasil): caracterização do acidente e da vítima. Rev. Saúde Públ. [Internet] 1985;19(5) [acesso 25 fev 2012]. Disponível: http://www.scielosp.org/pdf/ rsp/v19n5/11.pdf

15. PHTLS - Pre hospital trauma life support. NAEMT, 7th ed. Rio de Janeiro: Elsevier; 2011.

16. Credo PFD, Felix JVC. Perfil dos pacientes atendidos em um hospital de referência ao trauma em Curitiba: implicações para a enfermagem. Cogitare enferm. [Internet] 2012;17(1) ) [acesso 25 abr 2012]. Disponível: http://ojs.c3sl.ufpr.br/ojs2/index.php/cogitare/article/ view/26385/17578 
17. Guyton AC, Hall JE. Tratado de fisiologia médica. $12^{\mathrm{a}}$ ed. Rio de Janeiro: Elsevier; 2011.

18. Sessler DL. Complications and treatment of mild hypothermia. Anesthesiology, 2001;95(2):531-43. 